\title{
Dimercaptosuccinic acid scintigraphy vs. ultrasound for renal parenchymal defects in children
}

\author{
Maryse Marceau-Grimard, $M D^{\prime}$; Audrey Marion, MD'; Christian Côté, $M D^{2}$; Stephane Bolduc, $M D^{\prime}$; \\ Marcel Dumont, $M D^{2}$; Katherine Moore, $M D^{1}$
}

'Division of Urology, Department of Surgery; 2Division of Nuclear Medicine, Department of Medical Imaging; CHU de Québec, Université Laval, Quebec City, QC, Canada

Cite as: Can Urol Assoc J 2017;11 (8):260-4. hitp://dx.doi.org/10.5489/cuaj.4257

\section{Abstract}

Introduction: Dimercaptosuccinic acid (DMSA) scintigraphy is the gold standard in the evaluation of renal parenchymal defects and is widely used in the pediatric population. As more recent ultrasound equipment was purchased at our tertiary pediatric centre, our objective was to evaluate if renal ultrasound (US) results are equivalent or sufficient when compared to DMSA scintigraphy in the assessment of renal anomalies.

Methods: The charts of all 463 patients who underwent DMSA scintigraphy between January 2009 and May 2014 at our pediatric tertiary centre were reviewed. The objective was to look for correlation between US and DMSA scan results for renal scars/dysplasia. A hundred and sixty pediatric patients followed with US and DMSA scan for a total of 285 renal units remained for evaluation after exclusions. Timing of the exams, urinary tract infection (UTI), and indication for imaging were reviewed. Results with older (105 patients) and newer (55 patients) US equipment were compared. Results: Among the 285 renal units evaluated, 39 (14\%) had renal parenchymal defects shown by US and 87 (31\%) by DMSA scintigraphy (sensitivity $36 \%$, specificity $96 \%$ ). The DMSA scan was normal for eight abnormal kidneys (3\%) on US. The results were not statistically significant when compared to exams performed with newer or older US machines.

Conclusions: At our institution, US data are not sensitive enough to give reliable information about renal parenchymal defects, even with newer equipment. DMSA scintigraphy still remains mandatory for the evaluation of renal anomalies, but could be optional if the US exam indicates parenchymal defects.

\section{Introduction}

The gold standard imaging method to assess renal parenchymal defects (i.e., hypoplasia, dysplasia, scars), is considered to be dimercaptosuccinic acid (DMSA) scintigraphy. ${ }^{1}$ Even if it is well-tolerated, there are some concerns about accessibility, radiation exposure, and lengthy protocols in the pediatric population. ${ }^{2}$ Early detection of parenchymal dam- age is very useful in patient management, as it may lead from a conservative approach to surgical treatment. In addition, it helps determine the long-term prognosis and to establish appropriate followup. Acquired renal parenchymal anomalies are also a major risk factor for hypertension, proteinuria, pregnancy-related complications and, in severe cases, end-stage renal disease. ${ }^{2-5}$ Despite the debate regarding the most appropriate investigation, the least invasive imaging method providing sufficient clinical information is usually preferred. Recent advances in ultrasound (US) technology have led some physicians to believe that US study could now be more appropriate to evaluate renal defects. ${ }^{6-9}$ The constant improvement of imaging instruments, the increasing knowledge of users, and the increased awareness of the ALARA principle in urology made us reappraise our current practice. As more recent US equipment was purchased at our tertiary pediatric centre, we sought to evaluate whether we could solely rely on US compared to DMSA scintigraphy to assess renal parenchymal anomalies.

\section{Methods}

Our research's protocol was approved by the local research ethics board. We performed radiological and clinical retrospective chart review of all 463 children who underwent a DMSA scan at our centre between January 2009 and May 2014. One hundred and sixty patients (and 285 renal units) were included (Fig. 1). Selected pediatric patients had an US at our centre within 60 days of the DMSA scan without acute events between or at the time of the imaging process. We excluded from the analysis any anatomical abnormalities that could have invalidated the results, i.e., obesity, febrile urinary tract infection (UTI), and US performed without specific focus on the kidneys (Fig. 1). DMSA scintigraphy and US reports were independently reviewed and compared for each renal unit to create a realistic clinical scenario. DMSA scintigraphy was mostly performed for children with higher risk of renal parenchymal lesions, i.e., high-grade vesicoureteral reflux (VUR), recurrent febrile UTI, etc. 


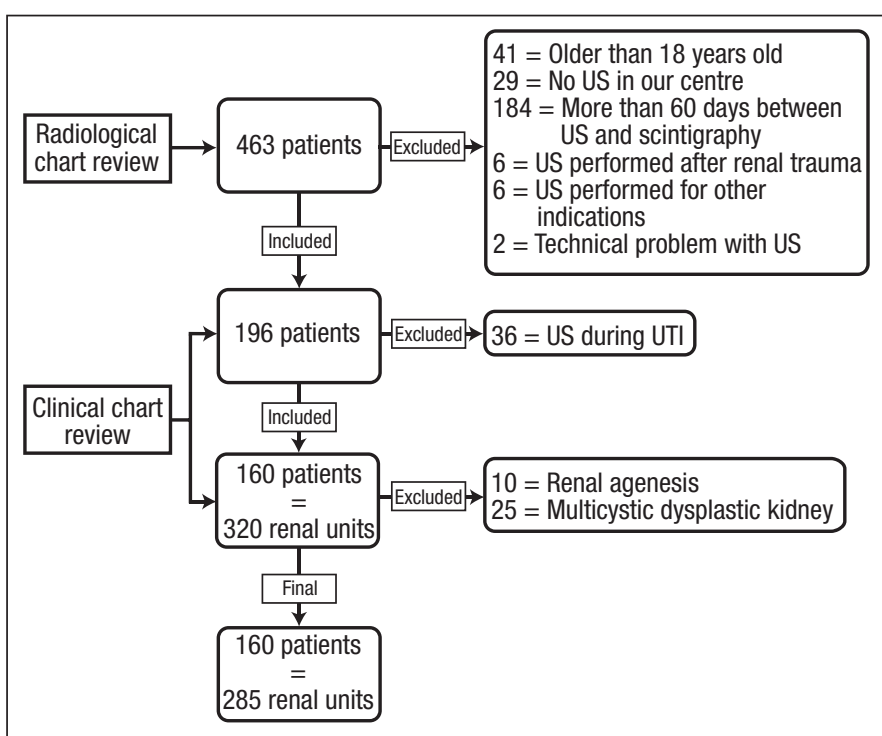

Fig. 1. Flow chart. US: ultrasound; UTI: urinary tract infection.

\section{DMSA scintigraphy}

All DMSA scans were performed in a standardized protocol. Each scintigraphy was interpreted by one of our two nuclear medicine pediatric specialists, who were unaware of the US results. Imaging was performed at least two hours following isotope administration using a dual detector gamma camera (Philips Axis) with a high-resolution, low-energy collimator for the delayed planar images and single-photon-emission computed tomography (SPECT). Three planar images of 500 kcounts on a $128 \times 128$ matrix format with adjustable zoom (1.5-3.2) were taken: posterior, left, and right posterior oblique. Relative renal function was evaluated on the basis of the posterior image after background correction. SPECT studies were sampled over 180 degrees on a $128 \times 128$ matrix with step and shoot, 65 second/step, total time 21 minutes. Iterative reconstruction was performed. Doses were scaled for patient weight (37 to $185 \mathrm{MBq}$ of 99 mTc-DMSA). No sedation was used. Report was considered abnormal when at least one of the following criteria was met, as in Patel et $\mathrm{al}^{10}$ : diffuse or sharp indentation in renal contour with thinning of cortex, any shaped defects with loss of renal volume, degree of photopenia or absent activity, and heterogeneous uptake of renal radionuclide. ${ }^{11}$ Defects located centrally over the pelvicalyceal system were not considered abnormal.

\section{US study}

Fourteen radiologists regularly rotate at our tertiary pediatric centre. They were unacquainted with other findings. US examinations were either performed using Philips/ATL HDI5000 (older, 105 patients) or IU-22 Philips (newer, 55

patients) (Philips Medical Systems, Andover, M, U.S.) with probes varying from $4-13 \mathrm{MHz}$, depending on the patient's size. A standardized US examination protocol was followed. Longitudinal and transverse grey-scale images through both kidneys, prone and supine, were obtained. Kidneys were routinely assessed for hydronephrosis, echogenicity, corticomedullary differentiation, lengths, and regularity of cortical outline. Doppler studies were not routinely performed.

Renal parenchymal defects on ultrasonography were defined as in Barry et al. ${ }^{12}$ This included approximation of sinus echoes to the cortical surface with or without underlying calyceal dilatation, irregularity of cortical outline, or a difference in prone renal length to denote parenchymal involvement on US. Underlying calyceal dilatation was not considered essential for the diagnosis of scarring or dysplasia.

\section{Statistics}

Data was analysed with MedCalc 12.3. Receiver operating characteristic (ROC) curves were obtained as in DeLong et al. ${ }^{13}$ The sensitivity, specificity, positive predictive value (PPV), and negative predictive value (NPV) of US in identifying renal parenchymal damage were calculated using DMSA scan as the reference method with contingency tables. Comparisons between US and DMSA scan findings were made using 95\% confidence interval $(\mathrm{Cl})$ of area under the curve $(A \cup C)$ analysis; $p \leq 0.05$ was considered significant. Difference between ROC curves for older and newer US machines was assessed with Hanley JA et al's method..$^{14,15}$

\section{Results}

Eighty-two males and 78 females were included for a total of 285 renal units (141 right and 144 left kidneys). Median age at the time of imaging studies was 1.1 year old (range 1 day -17 years) with a mean age of 2.2 years old. Main

\section{Table 1. Main indication for imaging studies}

\section{Indication}

Vesicoureteral reflux (VUR) (with or without UTI)*

Low-grade (I-III)

High-grade (IV-V)

Recurrent febrile urinary infection (without VUR)

Multicystic dysplastic kidney

Renal agenesis

Renal anomalies (ex: ureterocele, ectopic ureter, horseshoe kidney)

Hypertension

Ureteropelvic junction obstruction

Renal ectopy

"Patient with VUR and other coexistent conditions were classified in the VUR section. UTI: urinary tract infection. 


\begin{tabular}{lccc}
\hline \multicolumn{4}{l}{ Table 2. DMSA scan and US results by patients } \\
\hline Patients, $\mathbf{n}(\%)$ & Normal US & Abnormal US & Total \\
\hline Normal DMSA & $81(51)$ & $5(3)$ & $86(54)$ \\
Abnormal DMSA & $45(28)$ & $29(18)$ & $74(46)$ \\
Total & $126(79)$ & $34(21)$ & 160 \\
\hline DMSA: dimercaptosuccinic acid scintigraphy; US: ultrasound.
\end{tabular}

indications for imaging were high-grade VUR, recurrent febrile UTI, multicystic dysplastic kidney, renal agenesis, and hypertension (Table 1). One hundred and two (64\%) sets of exams were performed on the same day.

The US and DMSA scans results were recorded and classified by patient and by renal unit (Tables 2,3 ). Parenchymal involvement was seen on DMSA scans for 87 units (74 patients), but only 31 units (29 patients) were also identified by US. Relying solely on the US report, we would have missed radiological parenchymal defects in 56 renal units (28\% of all children); however, for eight renal units (five patients), no anomaly was seen on the DMSA scan, even though the US was positive for renal damage. The characteristics of patients with positive US and negative DMSA scan are listed in Table 4.

Overall, using DMSA scintigraphy as the gold standard, the sensitivity of older vs. newer US was $37 \%$ and $32 \%$, and the specificity was $98 \%$ vs. $93 \%$ (Table 5 ). The global sensitivity and specificity are $36 \%$ and $96 \%$, respectively. The PPVs of older and newer US were $88 \%$ and $64 \%$ and the NPVs were $77 \%$ vs $78 \%$, respectively (Table 5 ). The calculated AUC was between 0.63 and 0.68 for the different subgroups, with a $p$ value below 0.05 for all results presented (Fig. 2). A value of one means a perfect concordance between US and the gold standard, while 0.5 is as good as flipping a coin. The difference between the AUC of older and newer ultrasound machines was not statistically significant.

\section{Discussion}

The incidence of febrile UTI in healthy children is $1-3 \% .^{16-17}$ Subsequent renal parenchymal changes occur in $40 \%$ of those with VUR and $6 \%$ of those without VUR. ${ }^{18}$ Surgical management gains priority with regard to degree of parenchymal involvement. This is why renal screening should be performed with a high-sensitivity apparatus, especially since we now know that neither US nor DMSA is precise enough to detect VUR. ${ }^{19}$

US is commonly used because it is available at low cost and is radiation-free. DMSA scintigraphy is a secondary choice due to its radiation exposure and associated costs. Recent US equipment acquisition led us to presume that better sensitivity could be achieved. We were unable to observe a significant difference between the older and newer apparatus. We think that sensitivity was already maximal with the older instruments. We strongly believe that the lower sensitivity of US examination is due to the poorly
Table 3. DMSA scan and US results with older and newer US machines by renal units

\begin{tabular}{lccccc}
\hline Renal units, n (\%) & \multicolumn{2}{c}{ Normal US } & \multicolumn{2}{c}{ Abnormal US } & Total \\
\hline & Older & Newer & Older & Newer & \\
\hline Normal DMSA & $123(43)$ & $67(23,5)$ & $3(1)$ & $5(2)$ & $198(69.5)$ \\
Abnormal DMSA & $37(13)$ & $19(6,7)$ & $22(8)$ & $9(3)$ & $87(30.5)$ \\
Total & $160(56)$ & $86(30)$ & $25(9)$ & $14(5)$ & 285 \\
\hline
\end{tabular}

DMSA: dimercaptosuccinic acid scintigraphy; US: ultrasound.

defined criteria of parenchymal defects. Moreover, the PPV of newer US (64\%) was inferior to older US machines (88\%). The global PPV of US was $79 \%$. The low PPV could be explained by the low prevalence of renal scars in our population. Seventy-five percent of the positive US with negative DMSA scans were linked to renal atrophy (Table 4). Renal defects located centrally over the pelvicalyceal system were considered normal. There could also be ambiguous cases of renal atrophy with hydronephrosis, leading to normal reports. Unfortunately, there are no standard interpretations of neither DMSA or US. Systematic approaches to analyze DMSA scan and define renal scarring with US have been proposed, but they are not systematically used in clinic. ${ }^{10-12}$

Additionally, Levart et $\mathrm{al}^{8-9}$ concluded twice that US was sensitive enough to identify clinically significant scars. In their studies, US detected all severe renal parenchymal defects $(5 / 5,100 \%)$ seen on DMSA, with a lower sensitivity to detect moderate $(19 / 24,79.2 \%)$ and mild $(15 / 44,31.8 \%)$ defects. It remained unclear which renal parenchymal damage was clinically significant and how long the followup should be. To our knowledge, no correlation was established between the degree of parenchymal anomaly and the risk of long-term adverse events. Regardless of the size of the defect, the probability of hypertension was estimated by Silva et al to be $0 \%$ for patients without renal damage, $15 \%$ for patients with unilateral, and $45 \%$ for those with bilateral renal damage, defined by DMSA scan. ${ }^{20}$ In our study, we chose a binary approach instead of stratification because of the lack of precise description of renal anomaly severity on imaging reports.

\begin{tabular}{|c|c|c|}
\hline $\begin{array}{l}\text { Number of } \\
\text { patients }\end{array}$ & Reasons for imaging & Ultrasound findings \\
\hline 2 & VUR & Renal atrophy \\
\hline $1^{a}$ & UPJ obstruction & Renal atrophy \\
\hline 1 & Pyelonephritis & Non-specific renal lesion \\
\hline 1 & Renal anomalies & Fetal lobulation vs. renal scar \\
\hline $1^{\mathrm{a}}$ & UTI + PUV & Renal atrophy \\
\hline $1^{b}$ & Hypertension & Renal atrophy \\
\hline \multicolumn{3}{|c|}{$\begin{array}{l}\text { aThe other kidney had a positive DMSA scintigraphy; bboth kidneys positive on ultrasound } \\
\text { and both negative on DMSA scintigraphy. DMSA: dimercaptosuccinic acid scintigraphy; } \\
\text { PUV: posterior urethral valve; UPJ: ureteropelvic junction; US: ultrasound; UTI: urinary trac } \\
\text { infection; VUR: vesicoureteral reflux. }\end{array}$} \\
\hline
\end{tabular}




\begin{tabular}{lccccc}
\hline \multicolumn{1}{l}{ Table 5. Ultrasound compared to DMSA scan results } & & & \\
\hline & $\mathbf{n}$ & Sensitivity & Specificity & Positive predictive value & Negative predictive value \\
\hline Patients & 160 & 0.39 & 0.94 & 0.85 & 0.64 \\
Renal units (RU) & 285 & 0.36 & 0.96 & 0.79 & 0.77 \\
Older machines (RU) & 185 & 0.37 & 0.98 & 0.88 & 0.77 \\
Newer machines (RU) & 100 & 0.32 & 0.93 & 0.64 & 0.78 \\
\hline
\end{tabular}

DMSA: dimercaptosuccinic acid scintigraphy.

Furthermore, we used accessible information to the physician in a bona fide clinical setup to reflect the actual practice, thus avoiding non-reproducible research settings. For this reason, we relied on available radiologist reports, even if our pediatric urologists are used to reviewing US images. $5,7,9,12$ According to Barry et al, US could achieve excellent sensitivity and specificity to detect renal scars by dedicated pediatric radiologists using state-of-the-art equipment. ${ }^{12}$ Unfortunately, these circumstances did not reproduce the daily clinical scenario. ${ }^{7}$

We did not routinely performed Doppler studies because it was demonstrated that renal power Doppler US does not predict renal scarring after UTIs. ${ }^{21}$ Besides, the compared efficacy of DMSA and Doppler US determined that DMSA scintigraphy was the most sensitive method to detect renal anomalies. ${ }^{3}$

Another limitation is the wide range of expertise of our ultrasonographists, as commonly found in other centres. Roebuck et al's meta-analysis reported an interobserver reliability of $53-92 \%$ for DMSA. ${ }^{6}$ Our small sample size and the retrospective nature of our study may also explain the difficulty in showing a significant difference.

In addition, DMSA scintigraphy is a less-than-perfect gold standard, as shown on piglets with $85 \%$ sensitivity and $97 \%$ specificity for renal anomalies. ${ }^{22}$ Our estimated $36 \%$ sensitivity for US is far from ideal, since Roebuck et al concluded that the US evaluation could replace DMSA scintigraphy if its sensitivity was higher than $85 \% .{ }^{6}$ The same authors highlighted the need for studies with larger number of renal units to evaluate the accuracy of US in detecting renal defects. They identified 10 studies that contained sufficient information to calculate sensitivity and specificity of US relatively to DMSA scan. The discovered methodological flaws translated into a wide range of sensitivities (37-100\%) and specificities (65-99\%), indicating that the performance

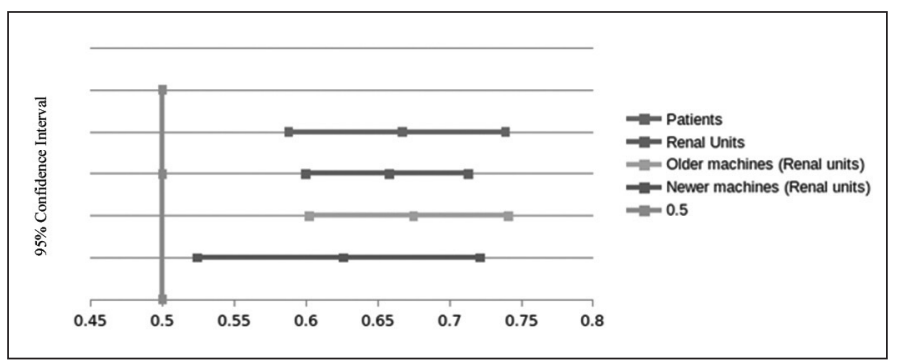

Fig. 2. Area under receiver operating characteristic (ROC) curve with a $95 \%$ confidence interval. of US remains controversial. ${ }^{6}$ As well, they concluded that DMSA scintigraphy was the best modality.

Given the high specificity of US $(96 \%)$ in our study, we argue that DMSA scintigraphy should be optional for children with an abnormal US. In a clinical scenario, the DMSA scan would not change the patient's management. In our cohort, 29 children (18\%) could have been exempted from the radiation exposure. But relying solely on US report, we would have missed radiological anomalies in $28 \%$ of all children. The sensitivity of US remains inferior to DMSA scan in the pediatric population. The NPV $(77 \%)$ shows that a negative US result is probably not as reliable as the DMSA scan. US should therefore not be considered a reference method for the evaluation of renal parenchymal defects.

\section{Conclusion}

At our centre, unique US studies, even with newer equipment, are not sensitive enough to detect renal anomalies. US could not be a substitute to DMSA scan. We consider US and DMSA scintigraphy to be complementary investigations in the assessment of children with possible renal parenchymal defects. The former provides structural information and the latter, functional details. Despite the poor sensitivity of US, its high specificity makes the DMSA scintigraphy optional when parenchymal anomaly is clearly detected. These findings confirm what actually occurs in our clinical practice.

Competing interests: Dr. Marceau-Grimard has received speaker honoraria from Pfizer. Dr. Bolduc has received clinical research grants from Astellas and Pfizer. Dr. Moore has been an advisor for Astellas and Pfizer; and has participated in clinical trials supported by Astellas, Ipsen, and Pfizer. The remaining authors report no competing personal or financial interests.

This paper has been peer-reviewed.

\section{References}

1. Bhatnagar V, Mitra DK, Agarwala S, et al. The role of DMSA scans in evaluation of the correlation between urinary tract infection, vesicoureteric reflux, and renal scarring. Pediatr Surg Int 2002;18:128-34. https://doi.org/10.1007/s003830100680

2. Temiz Y, Tarcan T, Onol FF, et al. The efficacy of Tc99m dimercatosuccinic acid (TC-DMSA) scintigraphy and ultrasonography in detecting renal scars in children with primary vesicoureteral relfux (VUR). Int Urol Nephrol 2006;38:149-52. https://doi.org/10.1007/s1 1255-005-3829-6 
3. Hitzel A, Liard A, Vera P, et al. Colour and power Doppler sonography vs. DMSA scintigraphy in acute pyelonephritis and in prediction of renal scaring. J Nucl Med 2002;43:27-32.

4. Ahmed M, Eggleston D, Kapur $G$, et al. Dimercaptosuccinic acid (DMSA) renal scan in the evaluation of hypertension in children. Pediatr Nephrol 2008;23:435-8. https://doi.org/10.1007/s00467-0070656-2

5. Shanon A, Feldman W, McDonald P, et al. Evaluation of renal scars by technetium-labeled dimercaptosuccinic acid scan, intravenous urography, and ultrasonography; a comparative study. J Pediatr 1992;120:399-403. https://doi.org/10.1016/S0022-3476(05)80904-7

6. Roebuck DJ, Howard RG, Metreweli C. How sensitive is ultrasound in the detection of renal scars? Br J Radiol 1999;72:345-8. htrps://doi.org/10.1259/bir.72.856.10474494

7. Moorthy I, Wheat D, Gordon I. Ultrasonography in the evaluation of renal scarring using DMSA scan as the gold standard. Pediatr Nephrol 2004;19:153-6. https://doi.org/10.1007/s00467-003-1363-2

8. Levart TK, Kenig A, Fettich JJ, et al. Sensitivity of ultrasonography in detecting renal parenchymal defects in children. Pediatr Nephrol 2002;17:1059-62. https://doi.org/10.1007/s00467-002-1007-y

9. Levart TK, Kliucewsek D, Kenig A, et al. Sensitivity of ultrasonography in detecting renal parenchymal defects: Six-year followup. Pediatr Nephrol 2009;24:1 193-7. https://doi.org/10.1007/s00467-0081099-0

10. Patel K, Charron M, Hoberman A, et al. Intra- and interobserver variability in interpretation of DMSA scans using a set of standardized criteria. Pediatr Radiol 1993;23:506-9. https://doi.org/10.1007/ BF02012131

11. Piepsz A, Blaufox MD, Gordon I, et al. Consensus on renal cortical scintigraphy in children with urinary tract infection. Scientific Committee of Radionuclides in Nephrourology. Semin Nucl Med 1999;29:160-74. https://doi.org/10.1016/S0001-2998(99)80006-3

12. Barry BP, Hall N, Cornford E, et al. Improved ultrasound detection of renal scarring in children following urinary tract infection. Clin Radiol 1998:53:747-51. https://doi.org/10.1016/S0009-9260(98)80317-6
13. DeLong ER, DeLong DM, Clarke-Pearson DL. Comparing the areas under two or more correlated receiver operating characteristic curves: A non-parametric approach. Biometrics 1988;44:837-45. https://doi.org/10.2307/2531595

14. Hanley JA, MCNeil BJ. The meaning and use of the area under a receiver operating characteristic (ROC) curve. Radiol 1982;143:29-36. https://doi.org/10.1148/radiology.143.1.7063747

15. VassarStats: Significance of the difference between the areas under two independent ROC curves. Inc.;c2001-16. Available at hittp://vassarstats.net/roc_comp.html. Accessed November 22, 2015.

16. Bykov S, Chervinsky L, Smolkin V, et al. Power Doppler sonography vs. TC-99m DMSA scintigraphy for diagnosing acute pyelonephritis in children: Are these two methods comparable? Clin Nucl Med 2003;28:198-203. https://doi.org/10.1097/01.RLU.0000053407.04034.B1

17. Clarke SE, Smellie JM, Prescod N, et al. Technetium-99m-DMSA studies in pediatric urinary infection. J Nucl Med 1996;37:823-8.

18. Polito C, Rambaldi PF, Signoriello $G$, et al. Permanent renal parenchymal defects after febrile UTI are closely associated with vesicoureteric reflux. Pediatr Nephrol 2006;21:521-6. https://doi.org/10.1007/ s00467-006-0036-3

19. Shaikh N, Spingarn RB, Hum SW. Dimercaptosuccinic acid scan or ultrasound in screening for wesicoureteral reflux among children with urinary tract infections. Cochrane Database Syst Rev 2016;7:CD010657.

20. Silva AC, Silva JM, Diniz JS, et al. Risk of hypertension in primary vesiocureteral reflux. Pediatr Nephrol 2007;22:459-6. htrps://doi.org/10.1007/s00467-006-0349-2

21. Narchi H, Donovan R. Renal power Doppler ultrasound does not predict renal scarring after urinary tract infection. Scott Med J 2008:53:7-10. https://doi.org/10.1258/RSMSMJ.53.4.7

22. Arnold AJ, Brownless SM, Carty HM, et al. Detection of renal scarring by DMSA scanning - an experimental study. J Pediatr Surg 1990;25:391-3. https://doi.org/10.1016/0022-3468(90)90377-L

Correspondence: Dr. Katherine Moore, Division of Urology, CHU de Québec, Université Laval, Quebec City, QC, Canada; katherine.moore.1@ulaval.ca

\section{COMING SOON!}

\section{CUA GUIDELINES \\ will be available in French}

\section{Topics include:}

- CUA guideline on the management of cystic renal lesions

- CUA guideline on adult overactive bladder

- CUA-PUC Canadian guidelines for the diagnosis, management and follow-up of cryptorchidism

The translations will be available

at the end of September at

$$
\text { cua.org and cuaj.ca }
$$

\section{À VENIR!}

\section{Publication en français des GUIDES DE PRATIQUE 2017 DE L'AUC}

\section{Thèmes abordés :}

- Guide de pratique sur la prise en charge des lésions kystiques aux reins

- Guide de pratique sur la vessie hyperactive chez l'adulte

- Guide de pratique AUC-UPC pour le diagnostic, la prise en charge et le suivi de la cryptorchidie au Canada

Les versions traduites seront affichées à la fin de septembre à cua.org et cuaj.ca 